\title{
The Global and Pullback Attractors for a Strongly Damped Wave Equation with Delays*
}

\author{
Guoguang Lin ${ }^{1}$, Fangfang Xia ${ }^{1}$, Guigui $\mathrm{Xu}^{2 \#}$ \\ ${ }^{1}$ Department of Mathematics, Yunnan University, Kunming, China \\ ${ }^{2}$ School of Mathematics and Science, Kaili University, Kaili, China \\ Email: gglin@ynu.edu.cn, \#xuguigui586@163.com
}

Received October 13, 2013; revised November 13, 2013; accepted November 21, 2013

Copyright (C) 2013 Guoguang Lin et al. This is an open access article distributed under the Creative Commons Attribution License, which permits unrestricted use, distribution, and reproduction in any medium, provided the original work is properly cited.

\begin{abstract}
In this paper, we study the global and pullback attractors for a strongly damped wave equation with delays when the force term belongs to different space. The results following from the solution generate a compact set.
\end{abstract}

Keywords: Strongly Damped; Pullback Attractor; Global Attractor; Delays

\section{Introduction}

Let $\Omega \subset \mathbb{R}^{\nVdash}$ be a bounded domain with smooth boundary $\partial \Omega$, we study the following initial boundary value problem

$$
\left\{\begin{aligned}
\frac{\partial^{2} u}{\partial t^{2}}+ & \alpha \frac{\partial u}{\partial t}-\beta \Delta \frac{\partial u}{\partial t}-\Delta u+g(u)=f(x)+h\left(t, u_{t}\right), \\
t & >\tau, \\
\left.u\right|_{\partial \Omega}= & 0, \\
t & \geq \tau-r, \\
u(x, t) & =\phi(x, t-\tau), \quad \frac{\partial u}{\partial t}(x, t)=\frac{\partial \phi}{\partial t}(x, t-\tau), \\
& x \in \Omega, t \in[\tau-r, \tau] .
\end{aligned}\right.
$$

where $f+h\left(t, u_{t}\right)$ is the source intensity which may depend on the history of the solution, $\alpha, \beta$ are the positive constants, $\phi$ is the initial value on the interval $[\tau-r, \tau]$ where $r>0$, and $u_{t}$ is defined for $\theta \in[-r, 0]$ as $u_{t}(\theta)=u(t+\theta)$. The assumption on $g(u)$ and $f(x)$ will be specified later.

It is well known that the long time behavior of many dynamical system generated by evolution equations can be described naturally in term of attractors of corresponding semigroups. Attractor is a basic concept in the study of the asymptotic behavior of solutions for the nonlinear evolution equations with various dissipation.

\footnotetext{
*This work is supported by the National Natural Sciences Foundation of People's Republic of China under Grant 11161057 and the planning project in 2013 of Kaili University under Grant Z1329.

\#Corresponding author.
}

There have been many researches on the long-time behavior of solutions to the nonlinear damped wave equations with delays. The existence of random attractors has been investigated by many authors, see, e.g., [1-4]. A new type of attractor, called a pullback attractor, was proposed and investigated for non-autonomous or these random dynamical systems. The pullback attractor describing this attractors to a component subset for a fixed parameter value is achieved by starting progressively earlier in time, that is, at parameter values that are carried forward to the fixed value. see [5-20]. However, to our knowledge, in the case of functional differential equations of second order in time, there is only partial results.

Recently, In [5], some results on pullback and forward attractor for the following strongly damped wave equation with delays

$$
\left\{\begin{aligned}
\frac{\partial^{2} u}{\partial t^{2}}+ & \alpha \frac{\partial u}{\partial t}-\Delta u=f(x)+h\left(t, u_{t}\right), \\
t & >\tau, \\
\left.u\right|_{\partial \Omega}= & 0, \\
t & \geq \tau-r, \\
u(x, t) & =\phi(x, t-\tau), \frac{\partial u}{\partial t}(x, t)=\frac{\partial \phi}{\partial t}(x, t-\tau), \\
& x \in \Omega, t \in[\tau-r, \tau] .
\end{aligned}\right.
$$

have been analyzed.

In this work, first, we apply the means in [3] to provide the existence of global attractor, for the dynamical system generated by the initial value problem 
(1.1). The key is to deal with the nonlinear terms and the delay term is difficult to be handled, so we aimed at showing that it is dissipative and the solution is bounded and continuous with respect to initial value. Hence we can discover the global attractor. Then, we aim to obtain the pullback attractor. The technology we use is introduced in [1], that is, we divide the semigroup into two: the one is asymptotically close to 0 , while the other is uniformly compact, so we can get the pullback attractor.

Now, we state the general assumptions for problem (1.1) on $g: \mathbb{R} \rightarrow \mathbb{R}$ and $h: \mathbb{R} \times C_{H} \rightarrow H$.

Let $G(u)=\int_{0}^{u} g(s) \mathrm{d} s$, then there exist positive constants $C_{i}(i=1,2, \cdots, 5)$ such that the followings hold true

$\left(\mathrm{G}_{1}\right) . \quad g(0) \in H$;

$\left(\mathrm{G}_{2}\right) . \quad\left|g^{\prime}(0)\right| \leq C_{1}$;

$\left(\mathrm{G}_{3}\right) .\left|g^{\prime \prime}(z)\right| \leq C_{2}\left(1+|z|^{\alpha}\right), \forall z \in \mathbb{R}$;

$\left(\mathrm{G}_{4}\right) . \liminf _{|z| \rightarrow \infty} g(z) / z \geq 0$;

(G). $(g(z)-g(0)) z \geq C_{4} z^{2}, \forall z \in \mathbb{R}$;

(G). $\liminf _{|z| \rightarrow \infty} \frac{g(z) z-C_{3} G(z)}{z^{2}} \geq 0$;

$\left(\mathrm{G}_{7}\right) . \quad-C_{5} \leq g^{\prime}(\mathrm{z}) \leq 0, \forall \mathrm{z} \in \mathbb{R}$.

For any $u \in V$, set $J(u)=\int_{\mathbb{R}^{3}} G(u) \mathrm{d} x$, by $\mathrm{G}_{1}-\mathrm{G}_{7}$, there are $C_{6} \geq 0, C_{\gamma} \geq 0$ and $\stackrel{\mathbb{R}}{\gamma}_{\gamma} \geq 0$, for any $\gamma>0$, we have

$$
\begin{aligned}
& (g(u) u, u)-C_{6} J(u) \geq-\gamma\|u\|^{2}-C_{\gamma} ; \\
& J(u) \geq-\gamma\|u\|^{2}-C_{\gamma} ; \\
& (g(u) u, u)-\rho_{\gamma}\|u\|^{2} \geq-\gamma\|\nabla u\|^{2}-C_{\gamma} .
\end{aligned}
$$

$\mathrm{H}_{1} . \forall \xi \in C_{H}, t \in \mathbb{R} \rightarrow h(t, \xi) \in H$ is continuous;

$\mathrm{H}_{2} . \forall t \in \mathbb{R}, h(t, 0)=0$;

$\mathrm{H}_{3} . \exists L_{h}>0$ such that $\forall t \in \mathbb{R}, \forall \xi, \eta \in C_{H}$

$$
|h(t, \xi)-h(t, \eta)| \leq L_{h}\|\xi-\eta\|_{C_{H}} ;
$$

$\mathrm{H}_{4} . \exists m_{0} \geq 0, C_{h}>0$ such that

$$
\forall m \in\left[0, m_{0}\right], \tau \leq t, u, v \in C^{0}([\tau-r, t] ; H)
$$

$\int_{\tau}^{t} e_{m s}\left|h\left(s, u_{s}\right)-h\left(s, v_{s}\right)\right|^{2} \mathrm{~d} s \leq C_{h}^{2} \int_{\tau-r}^{t} e_{m s}|u(s)-v(s)|^{2} \mathrm{~d} s ;$

$\mathrm{H}_{5} . h \in C^{1}\left(\mathbb{R} \times C_{H} ; H\right)$, and there exists $C>0$ such that, for any $(t, \xi) \in \mathbb{R} \times C_{H}$, the Frechet derivative $\delta h(t, \xi) \in \mathcal{L}\left(\mathbb{R} \times C_{H} ; H\right)$ satisfies

$$
\|\delta h(t, \xi)\|_{\mathcal{L}\left(\mathbb{R} \times C_{H} ; H\right)} \leq C\left(1+\|\xi\|_{C_{H}}\right) .
$$

The rest of this paper is organized as follows. In Section 2, we introduce basic concepts concerning global and pullback attractor. In Section 3, we obtain the existence of the global attractor. In Section 4, we obtain the existence of the pullback attractor.

\section{Preliminaries}

In this section,firstly, we recall some basic concepts about the global attractor.

Definition 2.1 ([3]) Let $X$ be a Banach space and $\{S(t)\}_{t \geq 0}$ be a family of operators on $X$. We say that $\{S(t)\}_{t \geq 0}$ is norm-to-weak continuous semigroup on $X$, if $\{S(t)\}_{t \geq 0}$ satisfies:

[1)] $S(0)=I d$ (identify) ;

[2)] $S(t) S(s)=S(t+s)$;

[3)] $S\left(t_{n}\right) x_{n} \rightarrow S(t) x$ if $t_{n} \rightarrow t \quad$ and $\quad x_{n} \rightarrow x \quad$ in $X$.

Remark: The strong continuous semigroup and the weak semigroup are both the norm-to-weak continuous

Definition 2.2 ([3]) The semigroup $S(t)_{t \geq 0}$ is called satisfying Condition (C) in $X$ if and only if for any bounded set $B$ of $X$ and for any $\epsilon>0$, there exist $a$ positive constant $t_{B}$ and a finite dimensional subspace $X_{1}$ of $X$, such that $\left\{P S(t) x \mid x \in B, t \geq t_{B}\right\}$ is bounded and

$$
\|(I-P) S(t) x\|_{X}<\epsilon \text { for any } t \geq t_{B} \text { and } x \in B,
$$

where $P: X \rightarrow X_{1}$ is the canonical projector.

Lemma 2.1 ([3]) Let $X$ be a Banach space and $\{S(t)\}_{t \geq 0}$ be a norm-to-weak continuous semigroup on $X$. Then $\{S(t)\}_{t \geq 0}$ has a global attractor in $X$ provided that the following conditions hold:

1) $\{S(t)\}_{t \geq 0}$ has a bounded absorbing set $B_{0}$ in $X$;

2) $\{S(t)\}_{t \geq 0}$ satisfies Condition (C) in $X$.

Then, we state the concepts and some result about the process and the pullback attractor.

Instead of a family of the one-parameter map $S(t)$, we need to use a two-parameter semigroup or process $U(t, \tau)$ on the complete metric space $X, u(t, \tau) \psi$ denotes the value of the solution at time $t$ which was equal to the initial value $\psi$ at time $\tau$.

The semigroup property is replaced by the process composition property

$$
U(t, \tau) U(\tau, r)=U(t, r) \text { for all } t \geq \tau \geq r,
$$

and, obviously, the initial condition implies $U(\tau, \tau)=I d$.

Definition 2.3 Let $U$ be the two-parameter semigroup or process on the complete metric space $X$. A family of compact set $\{\mathcal{A}(t)\}_{t \in \mathbb{R}}$ is said to be a pullback attractor for $U$ if, for all $\tau \in \mathbb{R}$, it satisfies

[1)] $U(t, \tau) \mathcal{A}(\tau)=\mathcal{A}(\tau)$ for all $t \geq \tau$, and

[2)] $\lim _{\operatorname{dist}_{X}}(U(t, t-s) \mathcal{D}, \mathcal{A}(t))=0 \quad$, for $\quad$ all bounded ${ }^{s} \overrightarrow{\mathcal{D}}^{\infty} \subset X$, and all $t \in \mathbb{R}$. 
Definition 2.4 The family $\{B(t)\}_{t \in \mathbb{R}}$ is said to be

1) pullback absorbing with respect to the process $U$, if for all $t \in \mathbb{R}$ and all bounded $D \subset X$, there exists $T_{D}(t)>0$ such that $U(t, t-s) D \subset B(t)$ for all $s \geq T_{D}(t)$;

2) pullback attracting with respect to the process $U$, if for all $t \in \mathbb{R}$, all bounded $D \subset X$, and all $\epsilon>0$, there exists $T_{\epsilon, D}(t)>0$ such that for all $s \geq T_{\epsilon, D}(t)$

$$
\operatorname{dist}_{X}(U(t, t-s) D, B(t))<\epsilon ;
$$

3) pullback uniformly absorbing (respectively uniformly attracting) if $T_{D}(t)$ in pact (a) (respectively $T_{\epsilon, D}(t)$ in part (b)) does not depend on the time $t$.

Theorem 2.1 Let $U(t, \tau)$ be a two-parameter process, and suppose $U(t, \tau): X \rightarrow X$ is continuous for all $t \geq \tau$. If there exists a family of compact pullback attracting sets $\{B(t)\}_{t \in \mathbb{R}}$, then there exists a pullback attractor $\{\mathcal{A}(t)\}_{t \in \mathbb{R}}$, such that $\mathcal{A}(t) \subset\{B(t)\}$ for all $t \in \mathbb{R}$, and which is given by

$$
\mathcal{A}=\overline{\bigcup_{D \subset X} \Lambda_{D}(t)} \text {, where } \Lambda_{D}(t)=\bigcap_{n \in \mathbb{N} s \geq n} \bar{U} U(t, t-s) D .
$$

We set $E=V \times H$, where $V=H_{0}^{1}(\Omega), H=L^{2}(\Omega)$, which are Hilbert spaces for the usual inner product and associated norms. we denote by $\lambda_{1}$ the first eigenvalue of $-\Delta$ in $V$.

Our problem can be written as a second-order differential equation in $H$ :

$$
\left\{\begin{array}{l}
u^{\prime \prime}+\alpha u^{\prime}-\beta \Delta u^{\prime}-\Delta u+g(u)=f(x)+h\left(t, u_{t}\right), t>\tau, \\
u(t)=\phi(t-\tau), \quad u^{\prime}(t)=\phi^{\prime}(t-\tau), \quad t \in[\tau-r, \tau]
\end{array}\right.
$$

\section{Existence of the Global Attractor}

In this section, our objection is to show that the well-posed of the solution and the existence of global attractor for the initial boundary value problem (1.1), we assume that $f \in L^{2}(\Omega)$.

Let $0<\varepsilon \leq \min \left\{\frac{1}{\beta}, \frac{\alpha}{4}, \frac{\lambda_{1}}{2 \alpha}\right\}$ and $\alpha>0, \beta>0$, then by the transformation $v=u^{\prime}+\varepsilon u$. The initial boundary value problem (2.1) is equivalent to

$$
\begin{aligned}
& v^{\prime}+(\alpha-\varepsilon) v+\varepsilon(\varepsilon-\alpha) u \\
& -\beta \Delta v+(\beta \varepsilon-1) \Delta u+g(u) \\
& =f(x)+h\left(t, u_{t}\right), t>\tau,
\end{aligned}
$$

with the initial value conditions

$$
v(t)=\phi^{\prime}(t-\tau)+\varepsilon \phi(t-\tau), \quad t \in[\tau-r, \tau] .
$$

Theorem 3.1 Assume that the hypotheses on $g$ and $h$ hold for all $\left(u, u^{\prime}\right)^{\mathrm{T}} \in E$ and $f \in L^{2}(\Omega), \alpha, \beta$ are the positive constants. Then the initial boundary value problem (3.1) has the unique solution $(u, v)^{\mathrm{T}} \in E$ for all $t>\tau$.
Proof. Taking the inner product of the Equation (3.1) with $v$ in $H$, we find that

$$
\begin{aligned}
& \frac{1}{2} \frac{\mathrm{d}}{\mathrm{d} t}|v|^{2}+(\alpha-\varepsilon)(v, v)+\varepsilon(\varepsilon-\alpha)(u, v)+\beta\|v\|^{2} \\
& +\frac{1-\beta \varepsilon}{2} \frac{\mathrm{d}}{\mathrm{d} t}\|u\|^{2}+\varepsilon(1-\beta \varepsilon)\|u\|^{2}+(g(u), v) \\
& =(f(x), v)+\left(h\left(t, u_{t}\right), v\right) .
\end{aligned}
$$

Since $v=u^{\prime}(t)+\varepsilon u$ and $0<\varepsilon \leq \min \left\{\frac{1}{2 \beta}, \frac{\alpha}{4}, \frac{\lambda_{1}}{2 \alpha}\right\}$, we deal with the terms in (3.2) one by one as follows

$$
\begin{gathered}
(\alpha-\varepsilon)(v, v)=(\alpha-\varepsilon)|v|^{2} \geq \frac{3 \alpha}{4}|v|^{2} ; \\
\varepsilon(\varepsilon-\alpha)(u, v) \geq \frac{\varepsilon(\varepsilon-\alpha)}{\sqrt{\lambda_{1}}}\|u\||v| \geq-\frac{\alpha \varepsilon}{\sqrt{\lambda_{1}}}|| u \||v| \\
\geq-\frac{\varepsilon \alpha^{2}}{\lambda_{1}}|v|^{2}-\frac{\varepsilon}{4}\|u\|^{2} \geq-\frac{\varepsilon}{4}\|u\|^{2}-\frac{\alpha}{2}|v|^{2} ; \\
(g(u), v)=\frac{\mathrm{d}}{\mathrm{d} t} J(u)+\varepsilon(g(u), u) \\
\geq \frac{\mathrm{d}}{\mathrm{d} t} J(u)+\varepsilon C_{6} J(u)-\varepsilon \gamma\|u\|^{2}-C_{\gamma} \varepsilon ; \\
\left(h\left(t, u_{t}\right), v\right) \leq \frac{1}{\alpha}|h|^{2}+\frac{\alpha}{4}|v|^{2} ; \\
(f, v) \leq \frac{1}{\alpha}|f|^{2}+\frac{\alpha}{4}|v|^{2}
\end{gathered}
$$

By (3.3)-(3.7), it follows from that

$$
\begin{aligned}
& \frac{\mathrm{d}}{\mathrm{d} t}\left(|v|^{2}+(1-\beta \varepsilon)\|u\|^{2}+2 J(u)\right)+\left(\frac{\alpha}{2}-\frac{\varepsilon \alpha^{2}}{\lambda_{1}}+\beta \lambda_{1}\right)|v|^{2} \\
& +2\left(\varepsilon(1-\beta \varepsilon)-\frac{\varepsilon}{4}-\varepsilon \gamma\right)\|u\|^{2}+2 \varepsilon C_{6} J(u) \\
& \leq \frac{2}{\alpha}|h|^{2}+\frac{2}{\alpha}|f|^{2}+2 \varepsilon C_{\gamma} .
\end{aligned}
$$

Since $\varepsilon \leq \min \left\{\frac{1}{\beta}, \frac{\alpha}{4}, \frac{\lambda_{1}}{2 \alpha}\right\}$ and $0<\gamma<\frac{1}{4}-\frac{\beta \varepsilon}{2}$, this will imply $2\left(\varepsilon(1-\beta \varepsilon)-\frac{\varepsilon}{4}-\varepsilon \gamma\right)>\varepsilon(1-\beta \varepsilon)$, then we have

$$
\begin{aligned}
& \frac{\mathrm{d}}{\mathrm{d} t}\left(|v|^{2}+(1-\beta \varepsilon)\|u\|^{2}+2 J(u)\right) \\
& +2 \beta \lambda_{1}|v|^{2}+\varepsilon(1-\beta \varepsilon)\|u\|^{2}+2 \varepsilon C_{6} J(u) \\
& \leq \frac{2}{\alpha}|h|^{2}+\frac{2}{\alpha}|f|^{2}+2 \varepsilon C_{\gamma} .
\end{aligned}
$$

Set $C_{0}=\min \left\{2 \beta \lambda_{1}, \varepsilon, 2 \varepsilon C_{6}\right\}$, then (3.8) can be writ- 
ten as following

$$
\begin{aligned}
& \frac{\mathrm{d}}{\mathrm{d} t}\left(|v|^{2}+(1-\beta \varepsilon)\|u\|^{2}+2 J(u)\right) \\
& +C_{0}\left(|v|^{2}+(1-\beta \varepsilon)\|u\|^{2}+2 J(u)\right) \\
& \leq \frac{2}{\alpha}|h|^{2}+\frac{2}{\alpha}|f|^{2}+2 \varepsilon C_{\gamma} .
\end{aligned}
$$

As our assumptions ensure that

$-C_{0}+\frac{2 C_{h}^{2} \lambda_{1}^{-1}}{\alpha(1-\beta \varepsilon-2 \gamma)}<0$, then we can choose

$m \in\left(0, m_{0}\right)$ small enough such that

$m-C_{0}+\frac{2 C_{h}^{2} \lambda_{1}^{-1}}{\alpha(1-\beta \varepsilon-2 \gamma)}<0$. For this choice, we have

$$
\begin{aligned}
& \frac{\mathrm{d}}{\mathrm{d} t} \mathrm{e}^{m t}\left(|v|^{2}+(1-\beta \varepsilon)\|u\|^{2}+2 J(u)\right) \\
& =m \mathrm{e}^{m t}\left(|v|^{2}+(1-\beta \varepsilon)\|u\|^{2}+2 J(u)\right) \\
& \quad+\mathrm{e}^{m t} \frac{\mathrm{d}}{\mathrm{d} t}\left(|v|^{2}+(1-\beta \varepsilon)\|u\|^{2}+2 J(u)\right) .
\end{aligned}
$$

Hence, we can get the following inequality

$$
\begin{aligned}
& \frac{\mathrm{d}}{\mathrm{d} t} \mathrm{e}^{m t}\left(|v|^{2}+(1-\beta \varepsilon)\|u\|^{2}+2 J(u)\right) \\
& \leq\left(m-C_{0}\right) \mathrm{e}^{m t}\left(|v|^{2}+(1-\beta \varepsilon)\|u\|^{2}+2 J(u)\right) \\
& \quad+\frac{2}{\alpha} \mathrm{e}^{m t}|h|^{2}+\frac{2}{\alpha} \mathrm{e}^{m t}|f|^{2}+2 \varepsilon C_{\gamma} \mathrm{e}^{m t} .
\end{aligned}
$$

By integrating over the interval $[\tau, t]$, we deduce

$$
\begin{aligned}
& \mathrm{e}^{m t}\left(|v|^{2}+(1-\beta \varepsilon)\|u\|^{2}+2 J(u)\right) \\
& \leq \mathrm{e}^{m \tau}\left(|v|^{2}+(1-\beta \varepsilon)\|u\|^{2}+2 J(u)\right)+\left(m-C_{0}\right) \int_{\tau}^{t} \mathrm{e}^{m s}\left(|v|^{2}+(1-\beta \varepsilon)\|u\|^{2}+2 J(u)\right) \mathrm{d} s \\
&+\frac{2}{\alpha} \int_{\tau}^{t} \mathrm{e}^{m s}|h|^{2} \mathrm{~d} s+\frac{2}{\alpha} \int_{\tau}^{t} \mathrm{e}^{m s}|f|^{2} \mathrm{~d} s+2 \varepsilon C_{\gamma} \int_{\tau}^{t} \mathrm{e}^{m s} \mathrm{~d} s \\
& \leq \mathrm{e}^{m \tau}\left(|v|^{2}+(1-\beta \varepsilon)\|u\|^{2}+2 J(u)\right)+\left(m-C_{0}\right) \int_{\tau}^{t} \mathrm{e}^{m s}\left(|v|^{2}+(1-\beta \varepsilon)\|u\|^{2}+2 J(u)\right) \mathrm{d} s \\
&+\frac{2 C_{h}^{2} \lambda_{1}^{-1}}{\alpha} \int_{\tau-r}^{t}|u|^{2} \mathrm{~d} s+\frac{2}{m \alpha}|f|^{2}\left(\mathrm{e}^{m t}-\mathrm{e}^{m \tau}\right)+\frac{2 \varepsilon C_{\gamma}}{m}\left(\mathrm{e}^{m t}-\mathrm{e}^{m \tau}\right) \\
&= \mathrm{e}^{m \tau}\left(|v|^{2}+(1-\beta \varepsilon)\|u\|^{2}+2 J(u)\right)+\left(m-C_{0}\right) \int_{\tau}^{t} \mathrm{e}^{m s}\left(|v|^{2}+(1-\beta \varepsilon)\|u\|^{2}+2 J(u)\right) \mathrm{d} s \\
&+\frac{2 C_{h}^{2} \lambda_{1}^{-1}}{\alpha}\left(\int_{\tau}^{t} \mathrm{e}^{m s}|u|^{2} \mathrm{~d} s+\int_{\tau-r}^{\tau} \mathrm{e}^{m s}|u|^{2} \mathrm{~d} s\right)+\frac{2}{m \alpha}|f|^{2}\left(\mathrm{e}^{m t}-\mathrm{e}^{m \tau}\right)+\frac{2 \varepsilon C_{\gamma}}{m}\left(\mathrm{e}^{m t}-\mathrm{e}^{m \tau}\right) .
\end{aligned}
$$

Since

$$
J(u) \geq-\gamma\|u\|^{2}-C_{\gamma},
$$

So we can have

$$
\begin{aligned}
& |v|^{2}+(1-\beta \varepsilon)\|u\|^{2}+2 J(u) \\
& =|v|^{2}+(1-\beta \varepsilon-2 \gamma)\|u\|^{2}+2 J(u)+2 \gamma\|u\|^{2} \\
& \geq(1-\beta \varepsilon-2 \gamma)\|u\|^{2}+|v|^{2}-2 C_{\gamma} .
\end{aligned}
$$

Noticing $0<\gamma<\frac{1}{4}-\frac{\beta \varepsilon}{2}$, we obtain

$$
\|u\|^{2} \leq \frac{|v|^{2}+(1-\beta \varepsilon)\|u\|^{2}+2 J(u)}{1-\beta \varepsilon-2 \gamma}+\frac{2 C_{\gamma}}{1-\beta \varepsilon-2 \gamma} .
$$

In the Bounded set $D \subset C_{V, H}$, for any $u \in D$, there exists a constant $d$ such that

$$
|v|^{2}+(1-\beta \varepsilon)\|u\|^{2}+2 J(u) \leq d^{2} .
$$

(3.10)-(3.13) means that

$\frac{2 C_{h}^{2} \lambda_{1}^{-1}}{\alpha} \int_{\tau}^{t} \mathrm{e}^{m s}|u|^{2} \mathrm{~d} s$

$$
\begin{aligned}
\leq & \frac{2 C_{h}^{2} \lambda_{1}^{-1}}{\alpha(1-\beta \varepsilon-2 \gamma)} \int_{\tau}^{t} \mathrm{e}^{m s}\left(|v|^{2}+(1-\beta \varepsilon)\|u\|^{2}+2 J(u)\right) \mathrm{d} s \\
& +\frac{4 C_{h}^{2} \lambda_{1}^{-1} C_{\gamma}}{m \alpha(1-\beta \varepsilon-2 \gamma)}\left(\mathrm{e}^{m t}-\mathrm{e}^{m \tau}\right) ;
\end{aligned}
$$

$\frac{2 C_{h}^{2} \lambda_{1}^{-1}}{\alpha} \int_{\tau-r}^{\tau} \mathrm{e}^{m s}|u|^{2} \mathrm{~d} s$

$\leq \frac{2 C_{h}^{2} \lambda_{1}^{-1}}{\alpha(1-\beta \varepsilon-2 \gamma)} \int_{\tau-r}^{\tau} \mathrm{e}^{m s}\left(|v|^{2}+(1-\beta \varepsilon)\|u\|^{2}+2 J(u)\right) \mathrm{d} s$

$+\frac{4 C_{h}^{2} \lambda_{1}^{-1} C_{\gamma}}{m \alpha(1-\beta \varepsilon-2 \gamma)}\left(\mathrm{e}^{m \tau}-\mathrm{e}^{m(\tau-r)}\right)$

$\leq \frac{2 C_{h}^{2} \lambda_{1}^{-1} r d^{2}}{\alpha(1-\beta \varepsilon-2 \gamma)} \mathrm{e}^{m \tau}+\frac{4 C_{h}^{2} \lambda_{1}^{-1} C_{\gamma}}{m \alpha(1-\beta \varepsilon-2 \gamma)}\left(\mathrm{e}^{m \tau}-\mathrm{e}^{m(\tau-r)}\right)$.

Hence, by (3.12)-(3.14) and the choice of 


$$
\begin{aligned}
& m-C_{0}+\frac{2 C_{h}^{2} \lambda_{1}^{-1}}{\alpha(1-\beta \varepsilon-2 \gamma)}<0,(3.9) \text { can be rewritten } \\
& \mathrm{e}^{m t}\left(|v|^{2}+(1-\beta \varepsilon)\|u\|^{2}+2 J(u)\right) \\
& \leq \mathrm{e}^{m \tau} d^{2}+\left(m-C_{0}+\frac{2 C_{h}^{2} \lambda_{1}^{-1}}{\alpha(1-\beta \varepsilon-2 \gamma)}\right) \\
& \quad \times \int_{\tau}^{t} \mathrm{e}^{m s}\left(|v|^{2}+(1-\beta \varepsilon)\|u\|^{2}+2 J(u)\right) \mathrm{d} s \\
& \quad+\frac{2}{m \alpha}|f|^{2}\left(\mathrm{e}^{m t}-\mathrm{e}^{m \tau}\right)+\frac{4 C_{h}^{2} \lambda_{1}^{-1} C_{\gamma}}{m \alpha(1-\beta \varepsilon-2 \gamma)}\left(\mathrm{e}^{m \tau}-\mathrm{e}^{m(\tau-r)}\right) \\
& \quad+\frac{4 C_{h}^{2} \lambda_{1}^{-1} C_{\gamma}}{m \alpha(1-\beta \varepsilon-2 \gamma)}\left(\mathrm{e}^{m t}-\mathrm{e}^{m \tau}\right)+\frac{2 C_{h}^{2} \lambda_{1}^{-1} r d^{2}}{\alpha(1-\beta \varepsilon-2 \gamma)} \mathrm{e}^{m \tau} \\
& \leq \mathrm{e}^{m \tau} d^{2}\left(1+\frac{2 C_{h}^{2} \lambda_{1}^{-1} r}{\alpha(1-\beta \varepsilon-2 \gamma)}\right)+\frac{2}{m \alpha}|f|^{2}\left(\mathrm{e}^{m t}-\mathrm{e}^{m \tau}\right) \\
& \quad+\frac{4 C_{h}^{2} \lambda_{1}^{-1} C_{\gamma}}{m \alpha(1-\beta \varepsilon-2 \gamma)}\left(\mathrm{e}^{m t}-\mathrm{e}^{m \tau}\right) \\
& \quad+\frac{4 C_{h}^{2} \lambda_{1}^{-1} C_{\gamma}}{m \alpha(1-\beta \varepsilon-2 \gamma)}\left(\mathrm{e}^{m \tau}-\mathrm{e}^{m(\tau-r)}\right) .
\end{aligned}
$$

So we can get by (3.16)

$$
\begin{aligned}
& \left(|v|^{2}+(1-\beta \varepsilon)\|u\|^{2}+2 J(u)\right) \\
& \leq \mathrm{e}^{m \tau} \mathrm{e}^{-m t} d^{2}\left(1+\frac{2 C_{h}^{2} \lambda_{1}^{-1} r}{\alpha(1-\beta \varepsilon-2 \gamma)}\right) \\
& \quad+\frac{2}{m \alpha}|f|^{2}\left(1-\mathrm{e}^{m \tau} \mathrm{e}^{-m t}\right) \\
& +\frac{4 C_{h}^{2} \lambda_{1}^{-1} C_{\gamma}}{m \alpha(1-\beta \varepsilon-2 \gamma)}\left(1-\mathrm{e}^{m \tau} \mathrm{e}^{-m t}\right) \\
& +\frac{4 C_{h}^{2} \lambda_{1}^{-1} C_{\gamma}}{m \alpha(1-\beta \varepsilon-2 \gamma)}\left(\mathrm{e}^{m \tau} \mathrm{e}^{-m t}-\mathrm{e}^{m(\tau-r)} \mathrm{e}^{-m t}\right) .
\end{aligned}
$$

which implies,for $t>\tau$

$$
\begin{gathered}
\left(|v|^{2}+(1-\beta \varepsilon)\|u\|^{2}+2 J(u)\right) \\
\leq \mathrm{e}^{m \tau} \mathrm{e}^{-m t} d^{2}\left(1+\frac{2 C_{h}^{2} \lambda_{1}^{-1} r}{\alpha(1-\beta \varepsilon-2 \gamma)}\right) \\
\quad+\frac{2}{m \alpha}|f|^{2}+\frac{4 C_{h}^{2} \lambda_{1}^{-1} C_{\gamma}}{m \alpha(1-\beta \varepsilon-2 \gamma)} .
\end{gathered}
$$

If we denote

$$
\begin{aligned}
& \rho_{0}^{2}=\frac{2}{m \alpha}|f|^{2}+\frac{4 C_{h}^{2} \lambda_{1}^{-1} C_{\gamma}}{m \alpha(1-\beta \varepsilon-2 \gamma)}, \\
& {\widehat{\rho_{0}}}^{2}=1+\frac{2 C_{h}^{2} \lambda_{1}^{-1} r}{\alpha(1-\beta \varepsilon-2 \gamma)}
\end{aligned}
$$

then (3.17) yields that

$$
\begin{aligned}
& |v|^{2}+(1-\beta \varepsilon)\|u\|^{2}+2 J(u) \\
& \leq \rho_{0}^{2}+{\widehat{\rho_{0}}}^{2} d^{2} \mathrm{e}^{m(\tau-t)}, \quad \forall t>\tau .
\end{aligned}
$$

which means that the initial boundary value problem (3.1) has the solution $\left(u, u^{\prime}\right)^{\mathrm{T}} \in E$.

Now, we prove the uniqueness of the solution. Assume that $u(\cdot)=u(\cdot ; \tau, \phi)$ and $v(\cdot)=v(\cdot ; \tau, \psi)$ are the two solutions of the initial boundary value problem (3.1), $\phi, \psi$ are the corresponding initial value,we denote $w(\cdot)=u(\cdot)-v(\cdot)$. Therefore we have

$$
w^{\prime \prime}+\alpha w^{\prime}-\beta \Delta w-\Delta w+g(u)-g(v)=h\left(t, u_{t}\right)-h\left(t, v_{t}\right) \text {. }
$$

we take the inner product of the above equation with $w^{\prime}$ and we obtain

$$
\begin{aligned}
& \frac{1}{2} \frac{\mathrm{d}}{\mathrm{d} t}\left(\left|w^{\prime}\right|^{2}+\|w\|^{2}\right)+\alpha\left|w^{\prime}\right|^{2}+\beta\|w\|^{2}+\left(g(u)-g(v), w^{\prime}\right) \\
& =\left(h\left(t, u_{t}\right)-h\left(t, v_{t}\right), w^{\prime}\right) .
\end{aligned}
$$

Since

$$
\begin{gathered}
\left|\left(g(u)-g(v), w^{\prime}\right)\right| \leq C_{5}|w|\left|w^{\prime}\right| ; \\
2\left(h\left(t, u_{t}\right)-h\left(t, v_{t}\right), w^{\prime}\right) \leq\left|h\left(t, u_{t}\right)-h\left(t, v_{t}\right)\right|^{2}+\left|w^{\prime}\right|^{2} .
\end{gathered}
$$

So (3.20) can yields that

$$
\begin{aligned}
& \frac{\mathrm{d}}{\mathrm{d} t}\left(\left|w^{\prime}\right|^{2}+\|\left. w\right|^{2}\right) \\
& \leq 2 C_{5}|w \|| w^{\prime}|+| h\left(t, u_{t}\right)-\left.h\left(t, v_{t}\right)\right|^{2}+|w|^{2} \\
& \leq\left|h\left(t, u_{t}\right)-h\left(t, v_{t}\right)\right|^{2}+C_{7}\left(\left|w^{\prime}\right|^{2}+\left\|w^{\prime}\right\|^{2}\right) . \\
& \quad \int_{\tau}^{t}\left|h\left(t, u_{t}\right)-h\left(t, v_{t}\right)\right|^{2} \mathrm{~d} s \leq C_{h}^{2} \int_{\tau-r}^{\tau}|u-v|^{2} \mathrm{~d} s \\
& \leq \lambda_{1}^{-1} C_{h}^{2} r\|\phi-\psi\|_{C_{V, H}}^{2}+\lambda_{1}^{-1} C_{h}^{2} \int_{\tau}^{t}\|w\|^{2} \mathrm{~d} s .
\end{aligned}
$$

Integrating (3.21) over the interval $[\tau, t]$, we can get

$$
\begin{aligned}
& \left|w^{\prime}(t)\right|^{2}+\|w(t)\|^{2} \\
& \leq\left|w^{\prime}(\tau)\right|^{2}+\|w(\tau)\|^{2}+C_{7} \int_{\tau}^{t}\left(\left|w^{\prime}\right|^{2}+\|w\|^{2}\right) \\
& \quad+\lambda_{1}^{-1} C_{h}^{2} r\|\phi-\psi\|_{C_{V, H}}^{2}+\lambda_{1}^{-1} C_{h}^{2} \int_{\tau}^{t}\|w\|^{2} \mathrm{~d} s \\
& \leq \\
& \quad\left(1+\lambda_{1}^{-1} C_{h}^{2} r\right)\|\phi-\psi\|_{C_{V, H}}^{2} \\
& \quad+\int_{\tau}^{t}\left(\left(\lambda_{1}^{-1} C_{h}^{2}+C_{7}\right)\|w\|^{2}+C_{7}\right)\left|w^{\prime}\right|^{2} \mathrm{~d} s .
\end{aligned}
$$

Set $\gamma_{1}=\max \left\{\lambda_{1}^{-1} C_{h}^{2}+C_{7}, C_{7}\right\}$, then we have

$$
\begin{aligned}
& \left|w^{\prime}(t)\right|^{2}+|w(t)|^{2} \\
& \leq\left(1+\lambda_{1}^{-1} C_{h}^{2} r\right)\|\phi-\psi\|_{V_{V, H}}^{2}+\gamma_{1} \int_{\tau}^{t}\left(\|w\|^{2}+\left|w^{\prime}\right|^{2}\right) \mathrm{d} s .
\end{aligned}
$$


Combining the Gronwall Lemma, we get

$$
\begin{aligned}
& \left|w^{\prime}(t)\right|^{2}+\|w(t)\|^{2} \\
& \leq\left(1+\lambda_{1}^{-1} C_{h}^{2} r\right)\|\phi-\psi\|_{C_{V, H}}^{2} e^{\gamma_{1}(t-\tau)} \text {, for all } t \geq \tau .
\end{aligned}
$$

If $\phi, \psi$ stand for the same initial value, there has

$$
\left|w^{\prime}(t)\right|^{2}+\|w(t)\|^{2} \leq 0 .
$$

that shows that

$$
\left|w^{\prime}(t)\right|^{2}=0, \quad\|w(t)\|^{2}=0 .
$$

that is

$$
w(t)=0 .
$$

therefore

$$
u=v .
$$

we get the uniqueness of the solution. So the proof of the theorem 3.1. has been completed.

By the theorem 3.1,we obtain the global smooth solution $\left(u, u^{\prime}\right)$ continuously depends on the initial value $\left(\phi, \phi^{\prime}\right)$, the initial boundary value problem (1.1) generates a continuous semigroup

$$
\{S(t)\}_{t \geq \tau}, S(t): E \rightarrow E ;\left(u, u^{\prime}\right)=S(t)\left(\phi, \phi^{\prime}\right) .
$$

Then $B_{\rho_{0}}=\left\{\left(u, u^{\prime}\right)\left\|\left(u, u^{\prime}\right)\right\|_{X} \leq \rho_{0}\right\}$ is a bounded absorbing set for the semigroup $\{S(t)\}_{t \geq \tau}$ generated by (1.1).

Under the assumption on $g$ and $f$, we can get the nonlinear term $g(u)$ is compact and continuous, $f(x)$ is continuous. Next, our object is to show that the $C^{0}$ semigroup $\{S(t)\}_{t \geq 0}$ satisfies cindition $C$.

Theorem 3.2 Assume that the hypotheses on $g$ and $h$ hold for all $\left(u, u^{\prime}\right)^{\mathrm{T}} \in E, \quad \alpha, \beta$ are positive constants. Then the $C^{0}$ semigroup $\{S(t)\}_{t \geq \tau}$ associated with initial value problem (3.1) satisfies cinditionC, that is, there exists $m \in \mathrm{N}$ and $T=T(B, R)$, for any $N \geq m, t \geq T$ such that

$\left|v_{2}\right|^{2}+(1-\beta \varepsilon)\left\|u_{2}\right\|^{2} \leq C, \quad C$ is the positive constant.

Proof. Let $\lambda_{j}$ be the eigenvalues of $-\Delta u$ and $w_{j}$ be the corresponding eigenvectors, $j=1,2, \cdots$, without loss of generality, we can assume that $\lambda_{1}<\lambda_{2}<\cdots$, and $\lim _{m \rightarrow \infty} \lambda_{m}=\infty$.

It is well known that $\left\{w_{j}\right\}_{j=1}^{\infty}$ form an orthogonal basis of $H_{0}^{1}$. We write

$$
H_{m}=\operatorname{span}\left\{w_{1}, w_{2}, \cdots, w_{m}\right\}
$$

Since $f \in H_{0}^{1}$ and $f$ is compact, for any $\varepsilon>0$, there exists some $m \in \mathbf{N}$ such that

$$
\left\|\left(I-P_{m}\right) f\right\| \leq \frac{\varepsilon}{2},
$$

$$
\left\|\left(I-P_{m}\right) g\right\| \leq \frac{\varepsilon}{2}, \text { for all } u \in B_{R}(0, R)
$$

where $P_{m}: H_{0}^{1} \rightarrow H_{m}$ is orthogonal projection and $R$ is the radius of the absorbing set. For any $\left(u, u_{t}\right) \in E$, we write

$$
\begin{aligned}
\left(u, u_{t}\right) & =\left(P_{m} u, P_{m} u_{t}\right)+\left(\left(I-P_{m}\right) u,\left(I-P_{m}\right) u_{t}\right) \\
& =\left(u_{1}, u_{1 t}\right)+\left(u_{2}, u_{2 t}\right) .
\end{aligned}
$$

We note that

$$
h_{2}=\left(I-P_{m}\right) h, g_{2}=\left(I-P_{m}\right) g, f_{2}=\left(I-P_{m}\right) f \text {, }
$$

Taking the inner product of the second equation of (3.1) with $v_{2}$ in $L^{2}(\mathcal{D})$, After a computation like in the proof of Theorem 3.1, we can yield that

$$
\begin{aligned}
& \frac{1}{2} \frac{\mathrm{d}}{\mathrm{d} t}\left(\left|v_{2}\right|^{2}+(1-\beta \varepsilon)\left\|u_{2}\right\|^{2}\right)+(\alpha-\varepsilon)\left|v_{2}\right|^{2} \\
& +\varepsilon(\varepsilon-\alpha)\left(u_{2}, v_{2}\right)+\beta\left\|v_{2}\right\|^{2} \\
& +\varepsilon(1-\beta \varepsilon)\left\|u_{2}\right\|^{2}+\left(g_{2}(u), v_{2}\right) \\
& =\left(f_{2}(x), v_{2}\right)+\left(h_{2}\left(t, u_{t}\right), v_{2}\right) .
\end{aligned}
$$

This is the same as in the proof of the Theorem 3.1, except for a replacement of $\lambda_{1}$ with $\lambda_{m+1}$. Combined with (3.23) , (3.24) and (3.4), then we have

$$
\begin{aligned}
& \frac{1}{2} \frac{\mathrm{d}}{\mathrm{d} t}\left(\left|v_{2}\right|^{2}+(1-\beta \varepsilon)\left\|u_{2}\right\|^{2}\right)+\left(\frac{3 \alpha}{4}+\beta \lambda_{m+1}-\frac{5 \varepsilon}{2}\right)\left|v_{2}\right|^{2} \\
& +\varepsilon(1-\beta \varepsilon)\left\|u_{2}\right\|^{2} \leq \frac{L_{h}^{2}}{2 \varepsilon}+\varepsilon^{2} .
\end{aligned}
$$

Choose $k_{\varepsilon}=\min \left\{\frac{3 \alpha}{4}+\beta \lambda_{m+1}-\frac{5 \varepsilon}{2}, 1\right\}$, we can get

$\frac{1}{2} \frac{\mathrm{d}}{\mathrm{d} t}\left(\left|v_{2}\right|^{2}+(1-\beta \varepsilon)\left\|u_{2}\right\|^{2}\right)+k_{\varepsilon}\left(\left|v_{2}\right|^{2}+\left\|u_{2}\right\|^{2}\right) \leq \frac{L_{h}^{2}}{2 \varepsilon}+\varepsilon^{2}$.

By Gronwall lemma, we can obtain

$$
\left|v_{2}\right|^{2}+(1-\beta \varepsilon)\left\|u_{2}\right\|^{2} \leq \frac{L_{h}^{2}+\varepsilon^{3}}{2 \varepsilon k_{\varepsilon}}
$$

for all $t>\tau, N \geq m$ and $\left(u, u^{\prime}\right) \in E$. This shows that Condition $C$ is satisfied, and the proof is completed.

Due to Lemma 2.1, Theorem 3.1 and Theorem 3.2, we obtain the following Theorem

Theorem 3.3 Assume that the hypotheses on $g$ and $h$ hold for all $\left(u, u^{\prime}\right)^{\mathrm{T}} \in E, \quad \alpha, \beta$ are positive constants. Then the $C^{0}$ semigroup $\{S(t)\}_{t \geq \tau}$ associated with initial value problem (3.1) has a global attractor in $E$.

\section{Existence of the Pullback Attractor}

In this subsection, we assume that $f \in H$, we aim to study the pullback attractor for the initial value problem 
(1.1).

From Theorem 3.1, the initial value problem (1.1) generates a family two-parameter semigroup $U(\cdot, \cdot)$ in $C_{V, H}$, which can be defined by

$$
U(t, \tau)(\phi)=u_{t}(\cdot ; \tau, \phi), \quad t \geq \tau, \phi \in C_{V, H}
$$

Lemma 4.1 Let $\phi, \psi$ be the two initial values for the problem (1.1), $\tau \in \mathbb{R}$ is the initial time, Denote by $u(\cdot)=u(\cdot ; \tau, \phi)$ and $v(\cdot)=v(\cdot ; \tau, \psi)$ the corresponding solutions to (1.1). Then, there exists a constant $\gamma_{1}>0$ which is independent of initial value value and time, such that the following estimates hold:

$$
\begin{aligned}
& \quad\left|u^{\prime}(t)-v^{\prime}(t)\right|^{2}+\|u(t)-v(t)\|^{2} \\
& \quad \leq\left(1+\lambda_{1}^{-1} C_{h}^{2} r\right)\|\phi-\psi\|_{C_{V, H}}^{2} \mathrm{e}^{\gamma_{1}(t-\tau)}, \text { for all } t \geq \tau ; \\
& \left\|u_{t}-v_{t}\right\|^{2} \\
& \leq\left(1+\lambda_{1}^{-1} C_{h}^{2} r\right)\|\phi-\psi\|_{V_{V, H}}^{2} \mathrm{e}^{\gamma_{1}(t-\tau)}, \text { for all } t \geq \tau+r .
\end{aligned}
$$

Proof. We denote $w=u-v$, by (3.22), we can get (4.1) easily.

If we consider $t \geq \tau+r$, then $t+\theta \geq \tau$ for any $\theta \in[-r, 0]$, and

$$
\begin{aligned}
& \left|w^{\prime}(t+\theta)\right|^{2}+\|w(t+\theta)\|^{2} \\
& \leq\left(1+\lambda_{1}^{-1} C_{h}^{2} r\right)\|\phi-\psi\|_{C_{V, H}}^{2} \mathrm{e}^{\gamma_{1}(t-\tau+\theta)} \\
& \leq\left(1+\lambda_{1}^{-1} C_{h}^{2} r\right)\|\phi-\psi\|_{C_{V, H}}^{2} \mathrm{e}^{\gamma_{1}(t-\tau)} .
\end{aligned}
$$

Thus, $\left\|w_{t}\right\|^{2} \leq\left(1+\lambda_{1}^{-1} C_{h}^{2} r\right)\|\phi-\psi\|_{C_{V, H}}^{2} \mathrm{e}^{\gamma_{1}(t-\tau)}, \forall t \geq \tau+r$.

Theorem 4.1 The mapping $U(t, \tau): C_{V, H} \rightarrow C_{V, H}$ is continuous for any $t \geq \tau$.

Proof. Let $\phi, \psi \in C_{V, H}$ be the initial value for the problem (1.1) and $t \geq \tau$. Denote by $u(\cdot)=u(\cdot ; \tau, \phi)$ and $v(\cdot)=v(\cdot ; \tau, \psi)$ the corresponding solutions to (1.1). Then, writing again $w=u-v$ we obtain the following. If $t \in[\tau-r, \tau]$, then $w(t)=\phi(t-\tau)-\psi(t-\tau)$ and

$$
\begin{aligned}
\left|w^{\prime}(t)\right|^{2}+|w(t)|^{2} & \leq\|\phi-\psi\|_{C_{V}}^{2}+\left\|\phi^{\prime}-\psi^{\prime}\right\|_{C_{H}}^{2} \\
& \leq\left(1+\lambda_{1}^{-1} C_{h}^{2} r\right)\|\phi-\psi\|_{C_{V, H}}^{2} \mathrm{e}^{\gamma_{1}(t-\tau+r)} .
\end{aligned}
$$

Thus, we have

$$
\begin{aligned}
& \left|w^{\prime}(t)\right|^{2}+\|w(t)\|^{2} \\
& \leq\left(1+\lambda_{1}^{-1} C_{h}^{2} r\right)\|\phi-\psi\|_{C_{V, H}}^{2} \mathrm{e}^{\gamma_{1}(t-\tau+r)}, \forall t \geq \tau-r,
\end{aligned}
$$

whence

$$
\left\|w_{t}\right\|^{2} \leq\left(1+\lambda_{1}^{-1} C_{h}^{2} r\right)\|\phi-\psi\|_{C_{V, H}}^{2} \mathrm{e}^{\gamma_{1}(t-\tau+r)}, \forall t \geq \tau,
$$

which implies the continuity of $U(t, \tau)$.

Theorem 4.2 Assume that the hypotheses on $g$ and $h$ hold with $m_{0}>0, \alpha, \beta$ are the positive constants.

Suppose in addition that $\sqrt{2} C_{h} \sqrt{\lambda_{1}}<\alpha_{1} \sqrt{1-2 \gamma}$. Then exists a family $\{B(t)\}_{t \in \mathbb{R}}$ of bounded sets in $C_{V, H}$ which is uniformly pullback absorbing fir the process $U(\cdot, \cdot)$. Moreover, $B(t)=B^{0}$ for all $t \in \mathbb{R}$, where $B^{0}$ is the bounded set in $C_{V, H}$.

Proof. By (3.18), we can have

$$
\begin{aligned}
& \|u(t ; \tau, \phi)\|^{2}+\left|u^{\prime}(t ; \tau, \phi)\right|^{2} \\
& \leq \rho_{0}^{2}+{\widehat{\rho_{0}}}^{2} d^{2} \mathrm{e}^{m(\tau-t)}, \quad \forall t \geq \tau .
\end{aligned}
$$

and, in particular,

$$
\|u(t ; \tau, \phi)\|^{2}+\left|u^{\prime}(t ; \tau, \phi)\right|^{2} \leq \rho_{0}^{2}+{\widehat{\rho_{0}}}^{2} d^{2}, \quad \forall t \geq \tau .
$$

Moreover, as $u(t ; \tau, \phi)=\phi(t-\tau)$ and $u^{\prime}(t ; \tau, \phi)=\phi^{\prime}(t-\tau)$ for $t \in[\tau-r, \tau]$, then inequality (4.3) holds true for $t \geq \tau-r$.

If we take now $t \geq \tau+r$, then for all $\theta \in[-r, 0]$ we have $t+\theta \geq \tau$ and so

$$
\|u(t ; \tau, \phi)\|^{2}+\left|u^{\prime}(t ; \tau, \phi)\right|^{2} \leq \rho_{0}^{2}+{\widehat{\rho_{0}}}^{2} d^{2} \mathrm{e}^{m(\tau-t)},
$$

or, in other words,

$$
\begin{aligned}
& \|U(t, \tau) \phi\|_{C_{V, H}}^{2} \\
& \leq \rho_{0}^{2}+{\widehat{\rho_{0}}}^{2} d^{2} \mathrm{e}^{m(\tau-t)}, \forall t \geq \tau+r, \phi \in D .
\end{aligned}
$$

Therefore, there exists $T_{D} \geq r$ such that

$$
\|U(t, t-s) \phi\|_{C_{V, H}}^{2} \leq \rho_{0}^{2}, \quad \forall t \in \mathbb{R}, s \geq T_{D}, \phi \in D .
$$

which means that the ball $B_{C_{V, H}}\left(0, \rho_{0}\right)=B^{0} \subset C_{V, H}$ is uniformly pullback absorbing for the process $U(\cdot, \cdot)$.

Remark: On the one hand, observe that if $t_{0} \in \mathbb{R}$ and $t \geq t_{0}$, then

$u\left(t+\theta ; t_{0}-s, \phi\right)=u\left(t+\theta ; t-\left(s+t-t_{0}\right), \phi\right)$ and $u^{\prime}\left(t+\theta ; t_{0}-s, \phi\right)=u^{\prime}\left(t+\theta ; t-\left(s+t-t_{0}\right), \phi\right)$ with $s+t-t_{0} \geq s$. As a sequence of (4.4) we have

$\left\|U\left(t, t_{0}-s\right) \phi\right\|_{C_{V, H}}^{2} \leq \rho_{0}^{2}, \quad \forall t_{0} \in \mathbb{R}, t \geq t_{0}, s \geq T_{D}, \phi \in D$.

or ,we have $\forall t_{0} \in \mathbb{R}, t \geq t_{0}, \theta \in[-r, 0], s \geq T_{D}, \phi \in D$

$$
\left\|u\left(t+\theta ; t_{0}-s, \phi\right)\right\|^{2}+\left|u^{\prime}\left(t+\theta ; t_{0}-s, \phi\right)\right|^{2} \leq \rho_{0}^{2} .
$$

On the other hand, (4.3) implies, $\forall t_{0} \in \mathbb{R}, t \geq t_{0}, s \in \mathbb{R}, t \geq t_{0}-s-r, \phi \in D$, $\left\|u\left(t ; t_{0}-s, \phi\right)\right\|^{2}+\left|u^{\prime}\left(t ; t_{0}-s, \phi\right)\right|^{2} \leq \rho_{0}^{2}+{\widehat{\rho_{0}}}^{2} d^{2}, \quad \forall t \geq \tau$.

Theorem 4.3 Under the assumption in Theorem 4.1. Then there exists a compact set $B^{2} \subset C_{V, H}$ which is uniformly pullback attracting for the process $U(\cdot, \cdot)$, and consequently, there exits the pullback attractor. 
$\{\mathcal{A}(t)\}_{t \in \mathbb{R}}$. Moreover, $\{\mathcal{A}(t)\}_{t \in \mathbb{R}} \subset C_{D(A), V}$ for all

$t \in \mathbb{R}$.

Proof. For each $\varepsilon \in \mathbb{R}$, the norm

$\|\phi\|_{\varepsilon}^{2}=\|\phi\|_{C_{V}}^{2}+\left\|\phi^{\prime}+\varepsilon \phi\right\|_{C_{H}}^{2}, \phi \in C_{V, H}$ is equivalent to

$\|\cdot\|_{0}:=\|\|_{C_{V, H}}$. This allows us to obtain absorbing ball for the original norm by proving the existence of absorbing balls for this new norm for some suitable value of $\varepsilon$. Indeed, let us denote $B_{\varepsilon}(0, \rho)=\left\{\phi \in C_{V, H}:\|\phi\|_{\varepsilon}<\rho\right\}$. Noticing that for $c_{1}=\max \left\{2,1+2 \varepsilon^{2} \lambda_{1}^{-1}\right\}$ it follows that

$$
\begin{aligned}
& \|\phi\|_{C_{V, H}}^{2}=\|\phi\|_{C_{V}}^{2}+\left\|\phi^{\prime}+\varepsilon \phi-\varepsilon \phi\right\|_{C_{H}}^{2} \\
& \leq\|\phi\|_{C_{V}}^{2}+2\left\|\phi^{\prime}+\varepsilon \phi\right\|_{C_{H}}^{2}+2 \varepsilon^{2}\|\phi\|_{C_{H}}^{2} \\
& \leq\left(1+2 \varepsilon^{2} \lambda_{1}^{-1}\right)\|\phi\|_{C_{V}}^{2}+2\left\|\phi^{\prime}+\varepsilon \phi\right\|_{C_{H}}^{2} \\
& \leq c_{1}\|\phi\|_{\varepsilon}^{2},
\end{aligned}
$$

we then have $B_{\varepsilon}(0, \rho) \subset B_{0}\left(0, c_{1}^{1 / 2} \rho\right)$.

Let $D \subset C_{V, H}$ be a bounded set, i.e. there exists $d>0$ such that for any $\phi \in D$ it holds

$$
\|\phi\|_{\varepsilon}^{2}=\|\phi\|_{C_{V}}^{2}+\left\|\phi^{\prime}+\varepsilon \phi\right\|_{C_{H}}^{2} \leq c_{1} d^{2} .
$$

Denote by $u(\cdot)=u(\cdot ; \tau, \phi)$ the solution of the problem (2.1), and consider the problems:

$$
\begin{aligned}
& \begin{cases}v^{\prime \prime}+\alpha v^{\prime}-\beta \Delta v^{\prime}-\Delta v+g(u)= & f(x)+h\left(t, u_{t}\right), t>\tau, \\
v(t)=0, \quad v^{\prime}(t)=0, & t \in[\tau-r, \tau] .\end{cases} \\
& \left\{\begin{array}{lc}
w^{\prime \prime}+\alpha w^{\prime}-\beta \Delta w^{\prime}-\Delta w=0, & t>\tau, \\
w(t)=\phi(t-\tau), \quad w^{\prime}(t)=\phi^{\prime}(t-\tau), & t \in[\tau-r, \tau] .
\end{array}\right.
\end{aligned}
$$

From the uniqueness of the solution of problems (2.1), (4.5) and (4.6) it follows that

$$
u(\cdot)=v(\cdot)+w(\cdot), \quad \forall \tau \in \mathbb{R} \text {, and } \forall t \geq \tau-r .
$$

Consequently, $U(t, \tau)$ can be written as

$$
\begin{gathered}
U(t, \tau)(\phi)=U_{1}(t, \tau)(\phi)+U_{2}(t, \tau)(\phi), \\
\forall \phi \in C_{V, H}, t \geq \tau-r .
\end{gathered}
$$

where $U_{1}(t, \tau)(\phi)=v_{t}(\cdot)=v_{t}(\cdot ; \tau, \phi)$ and $U_{2}(t, \tau)(\phi)=w_{t}(\cdot)=w_{t}(\cdot ; \tau, \phi)$ are the solutions of (4.5) and (4.6) respectively.

First, thanks to (4.4), but with $g=f=h-0$, it follows that

$$
\begin{aligned}
& \left\|w_{t}(\cdot ; \tau, \phi)\right\|_{C_{V}}^{2}+\left|w_{t}^{\prime}(\cdot ; \tau, \phi)\right|_{C_{V}}^{2} \\
& \leq c_{1} d^{2} \mathrm{e}^{m(r+\tau-t)}, \quad \forall t \geq \tau+r, \phi \in D .
\end{aligned}
$$

Furthermore, for $t_{0} \in \mathbb{R}, t \geq t_{0}$ and $s \geq T_{D} \geq r$,

$$
w\left(t ; t_{0}-s, \phi\right)=w\left(t ; t-\left(s-t_{0}+t\right), \phi\right) .
$$

with $s+t-t_{0} \geq s \geq T_{D} \geq r$. Thus, Equation (4.7) implies in particular

$$
\begin{gathered}
w\left(t ; t_{0}-s, \phi\right) \leq c_{1} d^{2} \mathrm{e}^{m\left(r+t_{0}-s-t\right)} \leq c_{1} d^{2} \mathrm{e}^{m(r-s)}, \\
\forall t_{0} \in \mathbb{R}, t \geq t_{0}, s \geq T_{D}, \phi \in D .
\end{gathered}
$$

Then we can obtain that

$$
\left\|U_{2}(t, t-s) \phi\right\|_{C_{V, H}}^{2} \leq c_{1} d^{2} \mathrm{e}^{m(r-s)}, \forall t \in \mathbb{R}, s \geq r, \phi \in D,
$$

whence,

$$
\lim _{s \rightarrow+\infty} \operatorname{supsup}_{t \in \mathbb{R}}\left\|U_{\phi \in D}(t, t-s) \phi\right\|_{C_{V, H}}^{2}=0 .
$$

Next, fix $t_{0} \in \mathbb{R}, s \geq T_{D}, \phi \in D$ and denote

$$
\begin{aligned}
u(t) & =u\left(t ; t_{0}-s, \phi\right), \\
v(t) & =v\left(t ; t_{0}-s, \phi\right), t \geq t_{0}-s-r, \\
F(t) & =f+h\left(t, u_{t}\right)-g, \quad t \geq t_{0}-s .
\end{aligned}
$$

Then, for $t \geq t_{0}$,

$$
\begin{aligned}
& |F(t)| \leq|f|+|g|+L_{h}\left\|u_{t}\right\| \\
& \leq|f|+|g|+L_{h} \lambda_{1}{ }^{-\frac{1}{2}} \rho_{0}=K_{1},
\end{aligned}
$$

and for $t \geq t_{0}-s$, we have

$$
\begin{aligned}
& |F(t)| \leq|f|+|g|+L_{h}\left\|u_{t}\right\| \\
& \leq|f|+|g|+L_{h} \lambda_{1}^{-\frac{1}{2}}\left(\rho_{0}^{2}+\hat{\rho}_{0}^{2} d^{2}\right)^{1 / 2} \\
& \leq K_{1}+L_{h} \lambda_{1}^{-\frac{1}{2}} \hat{\rho}_{0} d .
\end{aligned}
$$

Then, we deduce from the assumption on $h$ that $F^{\prime}(t)=\left\langle\delta h\left(t, u_{t}\right),\left(1+u_{t}^{\prime}\right)\right\rangle-\left\langle\delta g, u^{\prime}\right\rangle$ and $F^{\prime}(t) \leq K\left(1+\left\|u_{t}\right\|_{C_{H}}\right)\left(1+\left\|u_{t}^{\prime}\right\|_{C_{H}}\right)+C_{5}\left|u^{\prime}\right|$. Arguing as we did in order to obtain (4.8) and (4.9), we have

$$
\left|F^{\prime}(t)\right| \leq K\left(1+\lambda_{1}^{-\frac{1}{2}} \rho_{0}\right)\left(1+\rho_{0}\right)+C_{5} \rho_{0}=K_{2}, \quad \forall t \geq t_{0},
$$

and

$$
\begin{aligned}
F^{\prime}(t) \leq & K\left(1+\left(\rho_{0}^{2}+\hat{\rho}_{0}^{2} d^{2}\right)^{1 / 2}\right)\left(1+\lambda_{1}^{-\frac{1}{2}}\left(\rho_{0}^{2}+\hat{\rho}_{0}^{2} d^{2}\right)^{1 / 2}\right) \\
& +C_{5}\left(\rho_{0}^{2}+\hat{\rho}_{0}^{2} d^{2}\right)^{1 / 2}=K_{3}(d), \quad \forall t \geq t_{0}-s .
\end{aligned}
$$

Let us denote

$y(t)=\left\|v^{\prime}(t)+\frac{\alpha}{2} v(t)\right\|^{2}+|A v(t)-F(t)|^{2}$ and make use of the estimates in Theorem 4.2. On the one hand, for all $t \geq t_{0}-s$, 


$$
\begin{aligned}
& \frac{\mathrm{d}}{\mathrm{d} t}(y(t))+\frac{\alpha}{2} y(t) \leq \alpha|F(t)|^{2}+\frac{4}{\alpha}\left|F^{\prime}(t)\right|^{2}+\frac{\alpha^{3}}{8}\|v(t)\|^{2} \\
& \leq \alpha\left(K_{1}+L_{h} \lambda_{1}^{-\frac{1}{2}} \hat{\rho}_{0} d\right)^{2}+\frac{4}{\alpha} K_{3}(d)^{2}+\frac{\alpha^{3}}{8}\|v(t)\|^{2} .
\end{aligned}
$$

but, as (4.4) and (4.7) ensure

$$
\|v(t)\|^{2} \leq 2\|u(t)\|^{2}+2\|w(t)\|^{2} \leq 2 \rho_{0}^{2}+2 \hat{\rho}_{0}^{2} d^{2}+2 c_{1} d^{2} .
$$

if we denote by

$$
\begin{aligned}
K_{4}(d) & =\left(K_{1}+L_{h} \lambda_{1}^{-\frac{1}{2}} \hat{\rho}_{0} d\right)^{2} \\
& +\frac{4}{\alpha} K_{3}(d)^{2}+\frac{\alpha^{3}}{4}\left(\rho_{0}^{2}+\hat{\rho}_{0}^{2} d^{2}+c_{1} d^{2}\right) .
\end{aligned}
$$

then, in particular,

$$
y^{\prime}(t)+\frac{\alpha}{2} y(t) \leq K_{4} d, \forall t \in\left[t_{0}-s, t_{0}\right] .
$$

Noticing that $y\left(t_{0}-s\right)=\left|F\left(t_{0}-s\right)\right|^{2}$, the Gronwall lemma leads us to

$$
y\left(t_{0}\right) \leq \frac{2}{\alpha} K_{4}(d)+\left(K_{1}+L_{h} \lambda_{1}^{-\frac{1}{2}} \hat{\rho}_{0} d\right)^{2}=K_{5}(d) .
$$

On the other hand, if $t \geq t_{0}$, we deduce that

$$
\|v(t)\|^{2} \leq 2\|u(t)\|^{2}+2\|w(t)\|^{2} \leq 2 \rho_{0}^{2}+2 c_{1} d^{2} \mathrm{e}^{m(r-s)},
$$

and, from (4.8) and (4.10),

$$
\begin{aligned}
& y^{\prime}(t)+\frac{\alpha}{2} y(t) \leq \alpha K_{1}+\frac{4}{\alpha} K_{2}+\frac{\alpha^{3}}{8}\|v(t)\|^{2} \\
& \leq \alpha K_{1}+\frac{4}{\alpha} K_{2}+\frac{\alpha^{3}}{4} \rho_{0}^{2}+\frac{\alpha^{3}}{4} c_{1} d^{2} \mathrm{e}^{m(r-s)} \\
& =K_{6}+K_{7} d^{2} \mathrm{e}^{-m s}, \quad \forall t \geq t_{0} .
\end{aligned}
$$

Once again, the Gronwall lemma implies that

$$
\begin{aligned}
y(t) & \leq y\left(t_{0}\right) \mathrm{e}^{\frac{\alpha}{2}\left(t_{0}-t\right)}+\frac{2}{\alpha} K_{6}+\frac{2}{\alpha} K_{7} d^{2} \mathrm{e}^{-m s} \\
& \leq K_{5}(d) \mathrm{e}^{\frac{\alpha}{2}\left(t_{0}-t\right)}+\frac{2}{\alpha} K_{6}+\frac{2}{\alpha} K_{7} d^{2} \mathrm{e}^{-m s}, \quad \forall t \geq t_{0} .
\end{aligned}
$$

Then, there exists $T_{D}^{\prime} \geq T_{D}$ such that, if $s \geq T_{D}^{\prime}$,

$$
y(t) \leq K_{5}(d) \mathrm{e}^{\frac{\alpha}{2}\left(t_{0}-t\right)}+\frac{3}{\alpha} K_{6}, \forall t_{0} \in \mathbb{R}, t \geq t_{0} .
$$

Recalling that $y(t)=y\left(t ; t_{0}-s, \phi\right)$, if we fix $t \geq t_{0}$, take $s=T_{D}^{\prime}$ and denote $\tilde{s}=t-t_{0}+T_{D}^{\prime}$ we have, provided $t-t_{0}$ is large enough, that

$$
\begin{aligned}
y\left(t ; t_{0}-T_{D}^{\prime}, \phi\right) & =y\left(t ; t-\left(t-t_{0}+T_{D}^{\prime}\right), \phi\right) \\
& =y(t ; t-\tilde{s}, \phi) \leq \frac{4}{\alpha} K_{6} .
\end{aligned}
$$

In conclusion, there exists $T_{D}^{\prime \prime}>0$ such that for all $t \in \mathbb{R}$, and all $s \geq T_{D}^{\prime}+T_{D}^{\prime \prime}$,

$$
y(t ; t-s, \phi) \leq \frac{4}{\alpha} K_{6}, \quad \forall \phi \in D .
$$

Denoting $\hat{T}_{D_{A}}=T_{D}^{\prime}+T_{D}^{\prime \prime}+r$, we have for all $\phi \in D, t \in \mathbb{R}, s \geq \hat{T}_{D}$

$$
\begin{aligned}
& \left\|v^{\prime}(t ; t-s, \phi)+\frac{\alpha}{2} v(t ; t-s, \phi)\right\|^{2} \\
& +|A v(t ; t-s, \phi)-F(t ; t-s, \phi)|^{2} \leq \frac{4}{\alpha} K_{6},
\end{aligned}
$$

where

$$
F(t ; t-s, \phi)=f+h\left(t, u_{t}(t ; t-s, \phi)\right)-g(u(t ; t-s, \phi)) \text {. }
$$

But as for all $\phi \in D, t \in \mathbb{R}$ and $s \geq T_{D}$, we get $\|v(t ; t-s, \phi)\|_{2}^{2} \leq \rho_{0}^{2}$ and $|F(t ; t-s, \phi)|^{2} \leq K_{7}^{2}=2|f|^{2}+2 C_{5}^{2} \rho_{0}^{2}+2 L_{h} \lambda_{1}^{-1} \rho_{0}^{2}$, and, consequently, for all $\phi \in D, t \in \mathbb{R}$ and $s \geq T_{D}$,

$$
\begin{aligned}
& \left\|v^{\prime}(t ; t-s, \phi)\right\|^{2}+|A v(t ; t-s, \phi)|^{2} \\
& \leq \frac{4}{\alpha} K_{6} \leq \frac{8}{\alpha} K_{6}+\frac{\alpha^{2}}{2} \rho_{0}^{2}+2 K_{7}^{2},
\end{aligned}
$$

which shows that

$$
\left\|v_{t}(; t-s, \phi)\right\|_{C_{D(A), V}}^{2} \leq \rho_{1}^{2}=\frac{4}{\alpha} K_{6} \leq \frac{8}{\alpha} K_{6}+\frac{\alpha^{2}}{2} \rho_{0}^{2}+2 K_{7}^{2},
$$

for all $\phi \in D, t \in \mathbb{R}$ and $s \geq \hat{T}_{D}$. This means that the all $B^{1}=B_{C_{D(A), V}}\left(0, \rho_{1}\right)$ is the bounded set in $C_{D(A), V}$

which, in addition, is uniformly absorbing for the family of operators $U(\cdot, \cdot)$. As $B^{1}$ is the bounded set in $C_{D(A), V}$, then there exists $T_{B^{1}} \geq r$ such that

$$
U_{1}(t, t-s) B^{1} \subset B^{1}, \forall t \in \mathbb{R}, s \geq T_{B^{1}},
$$

and, therefore, the bounded set $B^{2} \subset C_{D(A), V}$ given

$$
B^{2}=\bigcup_{t \in \mathbb{R} s \geq T_{B^{1}}} U_{1}(t, t-s) B^{1} \subset B^{1},
$$

is uniformly pullback absorbing for $U_{1}(\cdot, \cdot)$ in $C_{V, \underline{H}}$.

By Ascoli-Arzelà theorem, we can prove that $\bar{B}^{2}$ is compact, so $\left\{B(t) \equiv \bar{B}^{2}\right\}_{t \in \mathbb{R}}$ is a family of compact subsets in $C_{V, H}$, which is also uniformly pullback attracting for $U(\cdot, \cdot)$, and the proof has been completed.

\section{REFERENCES}

[1] J. C. Robinson, "Infinite Dimensional Dynamical Systems,” Cambridge University Press, London, 2001. http://dx.doi.org/10.1007/978-94-010-0732-0

[2] R. Temam, "Infinite Dimensional Dynamical Systems in Mechanics and Physics," Springer-Verlag, New York, 1988. http://dx.doi.org/10.1007/978-1-4684-0313-8 
[3] C. K. Zhong, M. H. Yang and C. Y. Sun, "The Existence of Global Attractors for the Norm-to-Weak Continuous Semigroup,” Journal of Differential Equations, Vol. 223, No. 2, 2006, pp. 367-399.

[4] Y. Q. Xie and C. k. Zhong, "The Existence of Global Attractor for a Class of Nonlinear Evolution Equation," Journal of Applied Analysis, Vol. 336, No. 1, 2007, pp. 54-69. http://dx.doi.org/10.1016/j.jmaa.2006.03.086

[5] T. Caraballo, P. E. Kloeden and J. Real, "Pullback and Forward Attractor for a Damped Wave Equation with Delays," Stochastics and Dynamics, Vol. 4, No. 3, 2004, pp. 405-423. http://dx.doi.org/10.1142/S0219493704001139

[6] V. Pata and M. Squassina, "On the Strongly Damped Wave Equation," Communications in Mathematical Physics, Vol. 253, No. 3, 2005, pp. 511-533. http://dx.doi.org/10.1007/s00220-004-1233-1

[7] T. Caraballo and J. A. Langa, "On the Upper Semicontinuity of Cocycle Attractors for Non-Autonomous and Random Dynamical Systems,” Dynamics of Continuous Discrete and Impulsive Systems, Vol. 10, 2003, pp. 491513.

[8] T. Caraballo, P. Marin-Rubio and J. Valero, "Autonomous and Non-Autonomous Attractors for Differential Equations with Delays,” Journal of Differential Equations, Vol. 208, No. 1, 2005, pp. 9-41.

[9] T. Caraballo and J. Real, "Attractors for 2D-NavierStokes Models with Delays,” Journal of Differential Equations, Vol. 205, No. 2, 2004, pp. 270-296. http://dx.doi.org/10.1016/j.jde.2004.04.012

[10] D. Cheban, P. E. Kloeden and B. Schmalfuss, “The Relationship between Pullback, Forwards and Global Attractors of Nonautonomous Dynamical Systems," Nonlinear Dynamics and Systems Theory, Vol. 2, 2002, pp. 9-28.

[11] C. Y. Sun, S. h. Wang and C. K. Zhong, "Global Attractors for a Nonlassical Diffusion Equation," Acta Mathematica Sinica, English Series, Vol. 26B, No. 3, 2005, pp. 1-8.

[12] J. Hale, "Asymptotic Behavior of Dissipative Systems,"
American Mathematical Society, Providence, 1988.

[13] M. J. Garrido-Atienza and J. Real, "Existence and Uniqueness of Solutions for Delay Evolution Equations of Second Order in Time," Journal of Mathematical Analysis and Applications, Vol. 283, No. 2, 2003, pp. 582-609.

[14] M. H. Yang, J. Q. Duan and P. Kloeden, “Asymptotic Behavior of Solutions for Random Wave Equations with Nonliear Damping and White Noise,” Nonlinear Analysis: Real World Applications, Vol. 12, No. 1, 2011, pp. 464478. http://dx.doi.org/10.1016/j.nonrwa.2010.06.032

[15] T. Caraballo, G. Lukaszewicz and J. Real, "Pullback Attractors for Non-Autonomous 2D-Navier-Stokes Equations in Some Unbounded Domains," Comptes Rendus de l'Académie des Sciences, Vol. 342, No. 4, 2006, pp. 263268.

[16] Y. J. Wang, C. K. Zhong and S. F. Zhou, "Pullback Attractors for Non-Autonomous Dynamical Systems,” Discrete and Continuous Dynamical Systems, Vol. 16, 2006, pp. 587-614.

[17] Z. J. Yang, “Global Attractor for a Nonlinear Wave Equation Arising in Elastic Waveguide Model," Nonlinear Analysis: Theory, Methods \& Applications, Vol. 70, No. 5, 2009, pp. 2132-2142. http://dx.doi.org/10.1016/j.na.2008.02.114

[18] Z. J. Yan and X. Li, "Finite-Dimensional Attractors for the Kirchhoff Equation with a Strong Dissipation,” Journal of Mathematical Analysis and Applications, Vol. 375, No. 2, 2011, pp. 579-593. http://dx.doi.org/10.1016/j.jmaa.2010.09.051

[19] Y. Q. Xie and C. K. Zhong, "Asymptotic Behavior of a Class of Nonlinear Evolution Equations,” Nonlinear Analysis: Theory, Methods \& Applications, Vol. 71, No. 11, 2009, pp. 5095-5105. http://dx.doi.org/10.1016/j.na.2009.03.086

[20] S. B. Wang and G. W. Chen, "Cauchy Problem of the Generalized Double Dispersion Equation,” Nonlinear Analysis: Theory, Methods \& Applications, Vol. 64, No. 1, 2006, pp. 159-173. http://dx.doi.org/10.1016/j.na.2005.06.017 